\title{
Complement activation, placental malaria infection, and birth weight in areas characterized by unstable malaria transmission in central Sudan
}

\author{
Ammar Alim', Naser E.Bilall', Awad-Elkareem Abass', Elhassan M Elhassan², Ahmed A Mohmmed³ and Ishag Adam ${ }^{4 *}$
}

\begin{abstract}
Background: The pathogenesis of malaria during pregnancy is not completely understood. There are few published data on complement activation and malaria during pregnancy. This study aimed to investigate complement activation and malaria during pregnancy, and their association with hemoglobin and birth weight.

Methods: A cross-sectional study was conducted at Medani, Sudan. Soluble terminal complement complex (TCC) levels were measured using ELISA in maternal and cord blood samples from 126 parturient women.

Results: There were no Plasmodium falciparum-positive blood films from maternal peripheral blood, the placenta, or cord blood samples. Three (2.4\%) and $22(17.5 \%)$ of the placentas showed chronic and previous infection with histopathological examination, respectively, while 101 (80.2\%) of them had no malaria infection. The mean [SD] of the maternal (22.4 [6.1] vs. 26.5 [3.5] ng/ml, P<0.001) and cord blood (24.5 [4.5] vs. 26.8 [4.4] ng/ml, P = 0.024) TCC levels were significantly lower in cases of placental malaria infection $(n=25)$ than in those without placental malaria infection $(n=101)$. Linear regression showed that placental malaria infection was significantly associated with birth weight ( $-0.353 \mathrm{~g}, \mathrm{P}=0.013)$, but there were no associations between maternal and cord TCC levels and maternal hemoglobin, or between TCC levels and birth weight.
\end{abstract}

Conclusion: Maternal and cord blood TCC levels are lower in women with placental malaria infection than in those without placental malaria infection.

Virtual Slide: The virtual slide(s) for this article can be found here: http://www.diagnosticpathology.diagnomx.eu/vs/ 9600054761463915

Keywords: Malaria, Pregnancy, Complement, Hemoglobin, Birth weight, Sudan

\section{Background}

Malaria is a large public health problem in endemic tropical countries, especially sub-Saharan Africa. In subSaharan Africa, approximately 125 million pregnant women live in malaria-endemic areas and 32 million of these pregnant women are at risk of malaria [1,2]. Malaria during pregnancy can lead to adverse maternal and perinatal effects, mainly anemia and low birth weight (LBW) [3-5]. Pregnant Sudanese women are susceptible to malaria (even the severe form), regardless of their age and parity, and malaria is associated with anemia and LBW in these women [6-8].

\footnotetext{
* Correspondence: ishagadam@hotmail.com

${ }^{4}$ Faculty of Medicine, University of Khartoum, Khartoum, Sudan

Full list of author information is available at the end of the article
}

The exact mechanism by which malaria infection and placental inflammation result in fetal growth restriction and LBW is poorly understood. However, many chemokines and inflammatory cytokines are associated with malaria infection and malaria-related LBW [9]. The complement system is an essential part of innate immunity and the host defense, and it plays a role in induction of inflammation and initiation of acquired immune responses in malaria-infected individuals $[10,11]$. C5a affects angiogenesis and its levels are increased with placental malaria infection [12-14]. Anaphylatoxin C5a and one $\mathrm{C} 5 \mathrm{~b}$ molecule I are produced when the terminal pathway is activated if C3b forms a complex with C3convertases and generates $\mathrm{C} 5$-convertases, which can cleave $\mathrm{C} 5$. When $\mathrm{C} 5 \mathrm{~b}$ is associated with $\mathrm{C6}, \mathrm{C} 7, \mathrm{C} 8$, and 
multiple C9 molecules, this leads to the generation of C5b-9, which is also known as the membrane attack complex. Interestingly, generation of $\mathrm{C} 5 \mathrm{~b}$ does not only lead to formation of membrane attack complex, but the major part is diverted by control proteins (e.g., clusterin and $S$ protein) to form the C5b-9 complex, which is commonly referred to as the terminal complement complex (TCC) [15]. Recently, significantly higher levels of TCC were reported in women with Plasmodium falciparum placental malaria infection compared with the malaria-negative group [16]. Further investigation of the role of complement in the pathogenesis of malaria (and its effects) during pregnancy is necessary.

The current study was conducted in Medani Maternity Hospital, Central Sudan, to investigate TCC levels in women with placental malaria, and their effect on maternal hemoglobin and birth weight.

\section{Methods}

A cross-sectional study was conducted during August to December 2011 (the rainy and post-rainy season) in the labor ward of the Medani Maternity Hospital. The area of this study is characterized by unstable malaria transmission. $P$. falciparum is the main malaria parasite species in the area and transmission occurs during the rainy (July to September) and post-rainy season [17]. Medani Maternity Hospital is a referral tertiary hospital caring for women who receive antenatal care at the hospital or are referred from other health centers and hospitals, and women who live close to the hospital facility. High-risk pregnancies are referred to the hospital. However, many women without a high-risk pregnancy deliver at this hospital.

The total sample size was calculated to have over $80 \%$ power to detect a difference of $5 \%$ at $\alpha=0.05$. We assumed that $10 \%$ of women might not respond or have incomplete data.

After obtaining signed informed consent from the patients, information on socio-demographics, history of obstetrics, medical history, antennal attendance characteristics, and bed net use was gathered using structured questionnaires. Body mass index was calculated by measuring maternal weight and height, which was expressed as weight $(\mathrm{kg}) /$ height $(\mathrm{m})^{2}$. Newborns were weighed immediately following birth using the Salter scale and the sex of each newborn was recorded.

\section{Giemsa-stained blood smears for light microscopy}

Maternal, placental, and cord blood films were prepared. Slides were stained by $10 \%$ Giemsa and the number of asexual parasites was counted per 200 leukocytes, assuming a leukocyte count of 8000 leukocytes/ $\mu$ l (for thick films) or per 1000 red blood cells (for thin films). Blood films were considered negative if no parasites were detected in 100 oil immersion fields of a thick blood film, which was double-checked in a blind manner by an expert microscopist. Maternal hemoglobin concentrations were estimated by the HemoCue hemoglobinometer (HemoCue AB, Angelhom, Sweden).

The blood (maternal and cord) was then allowed to clot and centrifuged for 10 minutes at $3000 \mathrm{rpm}$ and the serum was separated and stored at $-20^{\circ} \mathrm{C}$ till the analyses.

\section{Placental histology}

The details of how placental histology was performed have been mentioned previously $[8,18,19]$. Briefly, a 3$\mathrm{cm}^{3}$ sample was obtained from the maternal surface approximately half the distance between the umbilical cord and the edge of the placenta. Each biopsy sample was immediately placed in 10\% neutral buffered formalin. Buffer was used to prevent formation of formalin pigment, which has similar optical characteristics and polarized light activity as malaria pigment [20]. All of the biopsy samples were stored at room temperature until histology was performed. The placental biopsy samples were then processed and were embedded in paraffin wax, by standard techniques. In every case, paraffin sections that were 4-mm thick were stained with hematoxylineosin and Giemsa stains. Placental malaria infection was characterized using histology as previously described by Bulmer et al. as follows [21]: uninfected (no parasites or pigment), acute (parasites in intervillous spaces), chronic (parasites in maternal erythrocytes and pigment in fibrin, or cells within fibrin and/or chorionic villous syncytiotrophoblast or strom), and previous (no parasites, and pigment confined to fibrin or cells within fibrin). The slides were examined by a pathologist who remained blind regarding the clinical characteristics of these samples.

\section{ELISA for measuring TCC levels}

Maternal and cord serum levels were measured using a human TCC ELISA kit (Biotain Pharma Co., Ltd., Xiamen City, Fujian Province, China) by following the manufacturer's protocol.

\section{Statistical analysis}

Data were entered into a computer using SPSS for windows (version 16.0). Continuous data (including TCC levels) were normally distributed and were compared between groups using Student's $t$ test. Multivariate analyses were performed using binary models for placental malaria infection as the dependent variable and linear models with hemoglobin, birth weight, and TCC (maternal and cord levels) levels as continuous dependent variables. Sociodemographic characteristics, education, antenatal care, residence, and placental malaria infections were the independent predictor of interest. Odds ratios (OR) and 95\% 
Table 1 Univariate and multivariate analyses of factors associated with placental malaria infection

\begin{tabular}{|c|c|c|c|c|c|c|}
\hline \multirow[b]{2}{*}{ Variable } & \multicolumn{3}{|c|}{ Univariate analysis } & \multicolumn{3}{|c|}{ Multivariate analysis } \\
\hline & \multicolumn{3}{|c|}{ OR $95 \% \mathrm{CI} P$} & \multicolumn{3}{|c|}{ OR $95 \% \mathrm{Cl} P$} \\
\hline Age & 0.9 & $0.9-1.0$ & 0.152 & 1.0 & $0.9-1.1$ & 0.524 \\
\hline Primiparae & 0.8 & $0.7-1.0$ & 0.185 & 1.2 & $0.3-4.0$ & 0.714 \\
\hline Residence & 0.6 & $0.3-1.4$ & 0.696 & 1.1 & $0.3-3.7$ & 0.785 \\
\hline Maternal education < secondary level & 0.7 & $0.2-1.8$ & 0.485 & 0.9 & $0.3-2.6$ & 0.850 \\
\hline lack of antenatal care & 1.3 & $0.6-2.5$ & 0.242 & 1.0 & $0.3-3.4$ & 0.920 \\
\hline Body mass index & 1.0 & $0.9-1.0$ & 0.668 & 1.1 & $0.8-1.3$ & 0.377 \\
\hline Hemoglobin & 0.3 & $0.1-0.8$ & 0.016 & 0.7 & $0.4-1.1$ & 0.201 \\
\hline Blood group $\mathrm{O}$ & & & & 0.9 & $0.3-2.5$ & 0.941 \\
\hline
\end{tabular}

confidence intervals $(\mathrm{CI})$ were calculated and a $\mathrm{P}$ value of $<0.05$ was considered significant.

\section{Ethics}

The study received ethical clearance from the Research Board at the Faculty of Medicine, University of Khartoum, Sudan.

\section{Results}

Out of the 126 women enrolled in the study, 51 $(41.5 \%)$ were primiparae and $62(49.2 \%)$ had rural residency. Two (1.6\%), 33 (26.2\%), and 91 (92.1\%) of these 126 women had no, one to two visits, and more than two visits of antenatal care, respectively. The majority $(123 ; 97.6 \%)$ of them used bed nets during the index pregnancy.

Thirty one (24.6\%) of these 126 women had blood group A, 17 (13.5\%) had blood group B, three (2.4\%) had blood group $\mathrm{AB}$, and 75 (59.5\%) had blood group $\mathrm{O}$. The mean (SD) hemoglobin level was 10.7 (1.1) g/dl, and 72 (57.1\%) of the women were anemic (hemoglobin $<11 \mathrm{~g} / \mathrm{dl}$ ).

Eleven $(8.7 \%)$ women delivered low-birth weight neonates $(<2500 \mathrm{~g})$.

\section{Malaria infections}

There were no $P$. falciparum-positive blood films from maternal peripheral blood, the placenta, or cord blood samples. Three (2.4\%) and $22(17.5 \%)$ of the placentas showed chronic and previous infection on a histopathological examination, respectively, while 101 (80.2\%) of them showed no signs of malaria infection. There were no significant associations between age, parity, residence, antenatal care, blood group, and placental malaria infection (Table 1).

Birth weight was significantly lower in women with placental malaria infection $(n=25)$ compared women who did not have placental malaria infection $(n=101$; 2929.2 [648.9] vs 3206.6 [485.3] g, $\mathrm{P}=0.023$ ). In linear regression, placental malaria infection was significantly associated with birth weight $(-0.353 \mathrm{~g}, \mathrm{P}=$ 0.013 , Table 2).

\section{Maternal and cord blood TCC levels}

The mean [SD] of the maternal (22.4 [6.1] vs. 26.5 [3.5], $\mathrm{P}<0.001)$ and cord (24.5 [4.5] vs. 26.8 [4.4], $\mathrm{P}=0.024)$ blood TCC levels were significantly lower in women with placental malaria infection $(n=25)$ than in those

Table 2 Linear regression analysis of factors associated with maternal hemoglobin and birth weight

\begin{tabular}{|c|c|c|c|c|c|c|}
\hline \multirow[b]{2}{*}{ Variable } & \multicolumn{3}{|c|}{ Maternal haemoglobin } & \multicolumn{3}{|c|}{ birth weight } \\
\hline & \multicolumn{3}{|c|}{ Coefficient SE P } & \multicolumn{3}{|c|}{ Coefficient SE P } \\
\hline Age & 11.1 & 1.4 & 0.291 & 0.033 & 0.013 & 0.014 \\
\hline Parity & -0.114 & 0.104 & 0.275 & -0.094 & 0.048 & 0.714 \\
\hline Residence & 0.005 & 0.271 & 0.985 & -0.013 & 0.126 & 0.920 \\
\hline Maternal education < secondary level & 0.289 & 0.126 & 0.252 & 0.047 & 0.121 & 0.720 \\
\hline lack of antenatal care & 0.266 & 0.272 & 0.330 & -0.106 & 0.129 & 0.414 \\
\hline Body mass index & -0.067 & 0.047 & 0.158 & 0.010 & 0.022 & 0.668 \\
\hline Blood group $O$ & -0.085 & 0.227 & 0.710 & 0.062 & 0.107 & 0.561 \\
\hline Placental malaria infection & -0.312 & 0.291 & 0.285 & -0.353 & 0.139 & 0.013 \\
\hline Hemoglobin & - & - & - & 0.051 & 0.044 & 0.247 \\
\hline Maternal terminal complement complex & 0.006 & 0.027 & 0.839 & 0.022 & 0.013 & 0.091 \\
\hline Cord terminal complement complex & -0.025 & 0.026 & 0.338 & -0.011 & 0.012 & 0.338 \\
\hline
\end{tabular}




\section{A}

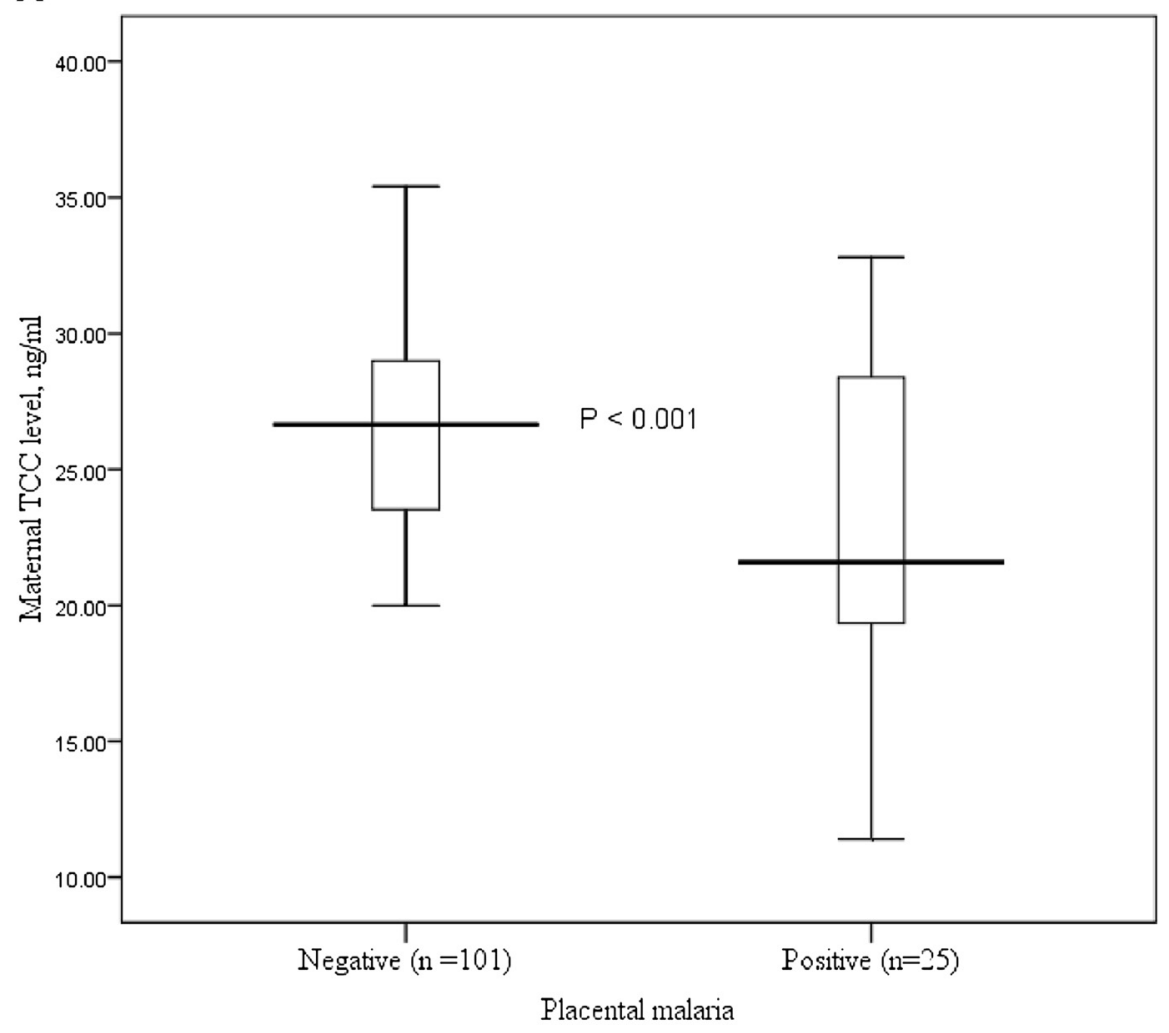

$\mathbf{B}$

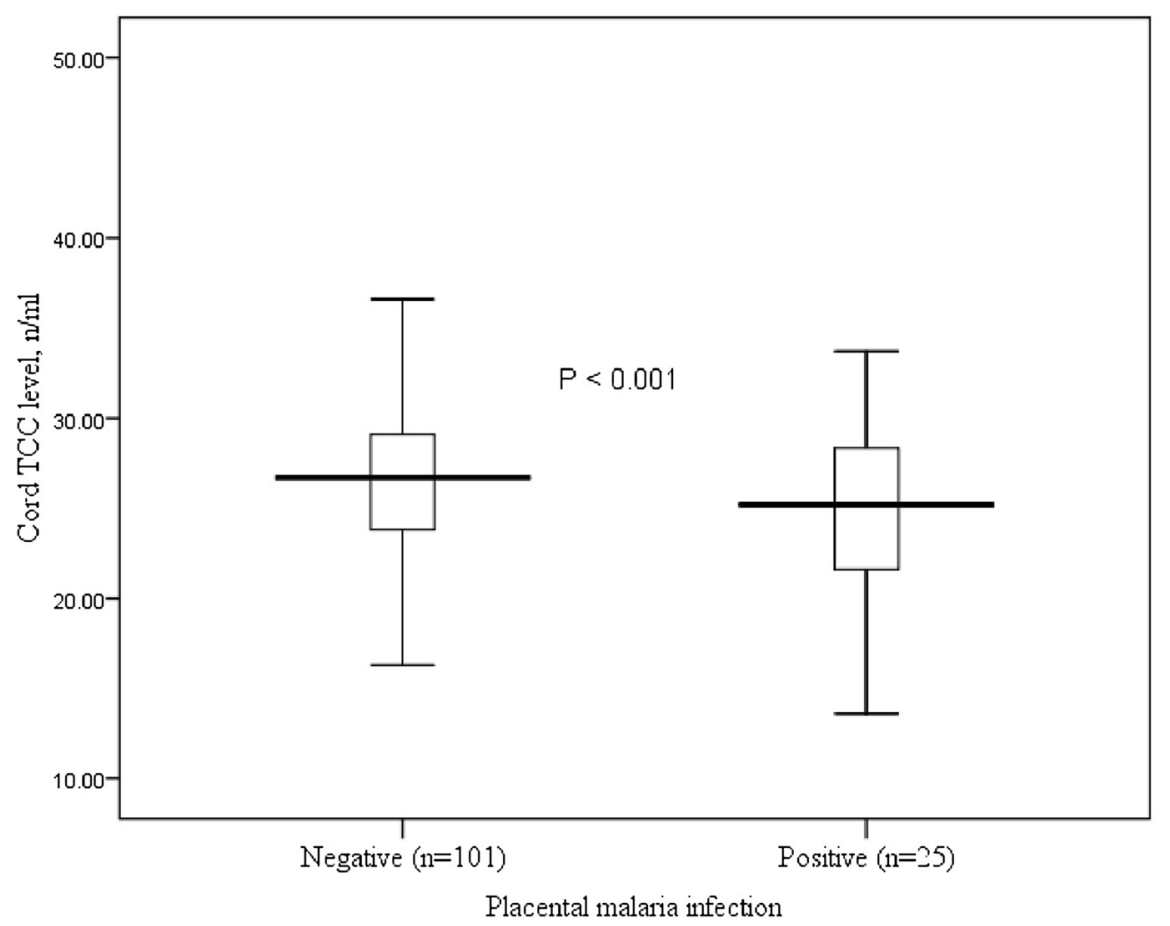

Figure 1 Box plots of TCC levels in maternal and cord blood of women with placental malaria and in those without malaria in central Sudan. 
without placental malaria infection $(n=101$, Figure 1$)$. Similarly, maternal (22.4 [6.1] vs. 26.5 [3.5], $\mathrm{P}<0.001)$ and cord (24.5 [4.5] vs. 26.8 [4.4], $\mathrm{P}=0.024$ ) blood TCC levels were significantly lower in women who had blood group $\mathrm{O}$ than in those who had blood groups other than $\mathrm{O}$.

In linear regression, placental malaria infection was associated with maternal $(-4.091 \mathrm{mg}, \mathrm{P}<0.001)$ and cord blood TCC levels $(-0.353 \mathrm{mg}, \mathrm{P}=0.013$, Table 3$)$.

There was no significant difference between maternal and cord blood TCC levels (25.7 [4.4] vs 26.3 [4.5], P = 0.802 ) in infected and non-infected women, even when the malaria-infected women were analyzed separately (22.5 [6.3] vs. 24.7 [6.4], $\mathrm{P}=0.138$ ).

\section{Discussion}

The main findings of the current study were that there were no associations between malaria placental infection and age or parity. Birth weight was significantly lower in newborns of malaria-infected women than in noninfected women. Furthermore, maternal and cord blood TCC levels were significantly lower in women with placental malaria infection than in those without placental malaria infection, and they had no effect on maternal hemoglobin levels and birth weight. The susceptibility of pregnant women to malaria infection, irrespective of their age or parity, is similar to previous findings in the same setting, as well as in different areas of Sudan $[6,8,18,19,22,23]$.

In the current study, maternal and cord blood TCC levels were significantly lower in women with placental malaria infection than in those without placental malaria infection. Bayoumi et al. observed that cytokine levels (interferon-gamma, interleukin-4, and interleukin-10) were significantly lower in peripheral and placental sera of women with placental malaria infection in eastern Sudan [23]. Recently, high C5a levels were observed in placental malaria infection [13,14]. Likewise, Khattab et al. reported significantly higher levels of TCC in women with $P$. falciparum placental malaria infection compared with the malaria-negative group [16]. Notably, different types of malaria were investigated between studies, where previous placental malaria infection (using histology) was observed in the current study and microscopically-detected malaria was observed in the later study. Furthermore, differences in malaria endemicity should be taken onto account when comparing studies because malaria had unstable transmission in the current study and it was hyperendemic in the second setting.

Interestingly, in this study, maternal and cord blood TCC levels were significantly lower in women with an $\mathrm{O}$ blood group compared with those with other blood types. Although there was no association between the $\mathrm{O}$ blood group and placental malaria infection in the current study, a previous study in eastern Sudan showed that women with blood type $\mathrm{O}$ were at higher risk of placental malaria infection $(\mathrm{OR}=1.9,95 \% \mathrm{CI}=1.1-3.2)$ [19]. However, recently, the reverse finding was observed where there was a lower prevalence of placental malaria in primiparae with blood group $\mathrm{O}$ [24]. Recently, Rowe and colleagues observed that blood group $\mathrm{O}$ protects against severe $P$. falciparum malaria by the mechanism of reduced resetting [25].

In the current study, although placental malaria infection was associated with maternal and cord blood TCC levels and birth weight, there was no association between either maternal or cord blood TCC levels and birth weight. Recent observations have shown that TCC levels are higher in placental plasma samples of newborns weighing less than $2700 \mathrm{~g}$ than in heavier newborns [16]. Submicroscopic placental malaria (which was not investigated in the current study), rather than histology-detected malaria, might be the main determinant of LBW in this setting [8].

\section{Conclusion}

There are significantly lower levels of maternal and cord blood TCC levels in women with placental malaria infection than in those without placental malaria infection. There is no association between maternal hemoglobin

Table 3 Linear regression analysis of factors associated with TCC

\begin{tabular}{|c|c|c|c|c|c|c|}
\hline \multirow[b]{2}{*}{ Variable } & \multicolumn{3}{|c|}{ Maternal TCC } & \multicolumn{3}{|c|}{ Cord TCC } \\
\hline & \multicolumn{3}{|c|}{ Coefficient SE P } & \multicolumn{3}{|c|}{ Coefficient SE P } \\
\hline Age & 0.147 & 0.084 & 0.084 & 0.033 & 0.013 & 0.014 \\
\hline Parity & 0.623 & 0.936 & 0.507 & -0.094 & 0.048 & 0.714 \\
\hline Body mass index & 0.056 & 0.166 & 0.736 & 0.010 & 0.022 & 0.668 \\
\hline Blood group $\mathrm{O}$ & -1.839 & 0.773 & 0.019 & 0.062 & 0.107 & 0.561 \\
\hline Placental malaria infection & -4.091 & 0.958 & $<0.001$ & -0.353 & 0.139 & 0.013 \\
\hline Hemoglobin & -0.025 & 0.340 & 0.941 & 0.051 & 0.044 & 0.247 \\
\hline Maternal terminal complement complex & - & - & - & 0.022 & 0.013 & 0.091 \\
\hline
\end{tabular}


and TCC (maternal and cord blood levels) levels or between TCC levels and birth weight.

\section{Competing interests}

The authors declare that they have no competing interests.

\section{Authors' contribution}

$A A$ and IA designed the study. NEB, AA and EME conducted the clinical work. AA and AAM conducted the laboratory lab. IA, NEB and EME performed the statistical analyses. All of the authors read and approved the final manuscript.

\section{Author details}

'Faculty of Medical laboratory Sciences, University of Khartoum, Khartoum, Sudan. ${ }^{2}$ Faculty of Medicine, University of Geziera, Medani, Sudan. ${ }^{3}$ Faculty of Medicine, Ribat University, Khartoum, Sudan. ${ }^{4}$ Faculty of Medicine, University of Khartoum, Khartoum, Sudan.

Received: 17 October 2014 Accepted: 16 April 2015

Published online: 06 May 2015

\section{References}

1. Dellicour S, Tatem AJ, Guerra CA, Snow RW, Ter Kuile FO. Quantifying the number of pregnancies at risk of malaria in 2007: a demographic study. PLoS Med. 2010;7:e1000221.

2. Desai M, ter Kuile FO, Nosten F, McGready R, Asamoa K, Brabin B, et al. Epidemiology and burden of malaria in pregnancy. Lancet Infect Dis. 2007;7:93-104

3. Menendez C, Ordi J, Ismail MR, Ventura PJ, Aponte JJ, Kahigwa E, et al. The impact of placental malaria on gestational age and birth weight. J Infect Dis. 2000;181:1740-5.

4. Rogerson SJ, Pollina E, Getachew A, Tadesse E, Lema VM, Molyneux ME. Placental monocyte infiltrates in response to Plasmodium falciparum malaria infection and their association with adverse pregnancy outcomes. Am J Trop Med Hyg. 2003;68:115-9.

5. Ahmed R, Singh N, ter Kuile FO, Bharti PK, Singh PP, Desai M, et al. Placental infections with histologically confirmed Plasmodium falciparum are associated with adverse birth outcomes in India: a cross-sectional study. Malar J. 2014;13:232.

6. Ali AA, Elhassan EM, Magzoub MM, Elbashir MI, Adam I. Hypoglycaemia and severe Plasmodium falciparum malaria among pregnant Sudanese women in an area characterized by unstable malaria transmission. Parasit Vectors. 2011;4:88.

7. Adam I, Khamis AH, Elbashir Ml. Prevalence and risk factors for anaemia in pregnant women of eastern Sudan. Trans R Soc Trop Med Hyg. 2005;99:739-43.

8. Mohammed AH, Salih MM, Elhassan EM, Mohmmed AA, Elzaki SE, El-Sayed BB, et al. Submicroscopic Plasmodium falciparum malaria and low birth weight in an area of unstable malaria transmission in Central Sudan. Malar J. 2013;12:172.

9. Umbers AJ, Aitken EH, Rogerson SJ. Malaria in pregnancy: small babies, big problem. Trends Parasitol. 2011;27:168-75.

10. Guo RF, Ward PA. Role of C5a in inflammatory responses. Annu Rev Immunol. 2005;23:821-52.

11. Silver KL, Higgins SJ, McDonald CR, Kain KC. Complement driven innate immune response to malaria: fuelling severe malarial diseases. Cell Microbiol. 2010:12:1036-45.

12. Langer HF, Chung KJ, Orlova W, Choi EY, Kaul S, Kruhlak MJ, et al. Complement-mediated inhibition of neovascularization reveals a point of convergence between innate immunity and angiogenesis. Blood. 2010;116:4395-403.

13. Conroy A, Serghides L, Finney C, Owino SO, Kumar S, Gowda DC, et al. C5a enhances dysregulated inflammatory and angiogenic responses to malaria in vitro: potential implications for placental malaria. PLoS One. 2009:4:e4953.

14. Muehlenbachs A, Fried M, Lachowitzer J, Mutabingwa TK, Duffy PE. Genome-wide expression analysis of placental malaria reveals features of lymphoid neogenesis during chronic infection. J Immunol. 2007;179:557-65.

15. Walport MJ. Complement. First of two parts. N Engl J Med. 2001;344:1058e66.

16. Khattab A, Kremsner PG, Meri S. Complement activation in primiparous women from a malaria endemic area is associated with reduced birth weight. Placenta. 2013;34:162-7.
17. Malik EM, Atta HY, Weis M, Lang A, Puta C, Lettenmaier C. Sudan Roll Back Malaria Consultative Mission: Essential Actions to Support the Attainment of the Abuja Targets. Sudan RBM Country Consultative Mission Final Report. Geneva: Roll Back Malaria Partnership; 2004.

18. Salih MM, Mohammed AH, Mohmmed AA, Adam GK, Elbashir Ml, Adam I. Monocytes and macrophages and placental malaria infections in an area of unstable malaria transmission in eastern Sudan. Diagn Pathol. 2011;6:83.

19. Adam I, Babiker S, Mohmmed AA, Salih MM, Prins MH, Zaki ZM. ABO blood group system and placental malaria in an area of unstable malaria transmission in eastern Sudan. Malar J. 2007;6:110.

20. Bulmer JN, Rasheed FN, Francis N, Morrison L, Greenwood BM. Placental malaria I. Pathological classification. Histopathology. 1993;22:211-8.

21. Bulmer JN, Rasheed FN, Morrison L, Francis N, Greenwood BM. Placental malaria II. A semi-quantitative investigation of the pathological features. Histopathology. 1993:22:219-25.

22. Batran SE, Salih MM, Elhassan EM, Mohmmed AA, Adam I. CD20, CD3, placental malaria infections and low birth weight in an area of unstable malaria transmission in Central Sudan. Diagn Pathol. 2013;8:189.

23. Bayoumi NK, Bakhet KH, Mohmmed AA, Eltom AM, Elbashir MI, Mavoungou E, et al. Cytokine profiles in peripheral, placental and cord blood in an area of unstable malaria transmission in eastern Sudan. J Trop Pediatr. 2009:55:233-7.

24. Bedu-Addo G, Gai PP, Meese S, Eggelte TA, Thangaraj K, Mockenhaupt FP. Reduced prevalence of placental malaria in primiparae with blood group $\mathrm{O}$. Malar J. 2014;13:289.

25. Rowe JA, Handel IG, Thera MA, Deans AM, Lyke KE, Koné A, et al. Blood group $\mathrm{O}$ protects against severe Plasmodium falciparum malaria through the mechanism of reduced rosetting. Proc Natl Acad Sci U S A. 2007;104:17471-6.

\section{Submit your next manuscript to BioMed Central and take full advantage of:}

- Convenient online submission

- Thorough peer review

- No space constraints or color figure charges

- Immediate publication on acceptance

- Inclusion in PubMed, CAS, Scopus and Google Scholar

- Research which is freely available for redistribution 